\title{
Opposite Adaptive Processing of Stimulus Intensity in Two Major Nuclei of the Somatosensory Brainstem
}

\author{
Boaz Mohar, Yonatan Katz, and Ilan Lampl \\ Department of Neurobiology, Weizmann Institute of Science, Rehovot 7610001, Israel
}

Tactile information ascends from the brainstem to the somatosensory cortex via two major parallel pathways, lemniscal and paralemniscal. In both pathways, and throughout all processing stations, adaptation effects are evident. Although parallel processing of sensory information is not unique to this system, the distinct information carried by these adaptive pathways remains unclear. Using in vivo intracellular recordings at their divergence point (brainstem trigeminal complex) in rats, we found opposite adaptation effects in the corresponding nuclei of these two pathways. Increasing the intensity of vibrissa stimulation entailed more adaption in paralemniscal neurons, whereas it caused less adaptation in lemniscal cells. Furthermore, increasing the intensity sharpens lemniscal receptive field profile as adaptation progresses. We hypothesize that these pathways evolved to operate optimally at different dynamic ranges of sustained sensory stimulation. Accordingly, the two pathways are likely to serve different functional roles in the transmission of weak and strong inputs. Hence, our results suggest that due to the disparity in the adaptation properties of two major parallel pathways in this system, high and reliable throughput of information can be achieved at a wider range of stimulation intensities than by each pathway alone.

\section{Introduction}

Sensory adaptation, the change of neural activity during sustained stimulation, is present in all sensory modalities and at different stages of processing (Dalton, 2000; Baccus and Meister, 2002; Heiss et al., 2008; Schweinberger et al., 2008). Adaptation has been implicated in different functional roles including, for example, gain control, predictive coding, and novelty detection (Ohzawa et al., 1982; Grill-Spector et al., 2006; for review, see Wark et al., 2007). While it is accepted that stimulus intensity is encoded by the magnitude of neuronal response (Wilent and Contreras, 2004; i.e., firing rate), due to adaptation, coding of intensity is inherently ambiguous. Accordingly, variations in neuronal activity may reflect fluctuations in the external stimulation but may also result from internal dynamics of adaptation. While reference to this ambiguity in the literature exists (Fairhall et al., 2001), so far it has not been sufficiently addressed.

In the somatosensory system, adaptation profoundly shapes the neuronal response along the pathways conveying tactile information to the cortex (Wang et al., 2010; Hansen and Dragoi, 2011). The major mechanism proposed for adaptation in this

Received April 27, 2013; revised July 12, 2013; accepted Aug. 14, 2013.

Author contributions: B.M. and I.L. designed research; B.M. performed research; B.M. and Y.K. analyzed data; B.M., Y.K., and I.L. wrote the paper.

This work was supported by Israel Science Foundation Grants 1160/11 and 1565/10; and by the Minerva Foundation, funded by the Federal German Ministry for Education and Research, ERA-Net Neuron, and Deutsche Forschungsgemeinschaft through the Collaborative Research Center Project 889 ("Cellular Mechanisms of Sensory Processing"). We thank Miriam Shafran and Adi Zarenkin for their help with the histological reconstructions; and Elad Ganmor, Shirley Mark, Aryeh Taub, and Inbal Meir for their helpful comments on this work.

The authors declare no competing financial interests.

Correspondence should be addressed to llan Lampl, Department of Neurobiology, Weizmann Institute of Science, Rehovot 7610001, Israel. E-mail: ilan.lampl@weizmann.ac.il.

DOI:10.1523/JNEUROSCI.1886-13.2013

Copyright $\odot 2013$ the authors $\quad 0270-6474 / 13 / 3315394-07 \$ 15.00 / 0$ system is short-term synaptic depression (STD; Tsodyks and Markram, 1997; Chung et al., 2002), in which the response gradually adapts due to the limited synaptic resources and their slow recovery. STD predicts more adaptation with increasing stimulation intensity due to increased presynaptic firing probability and therefore a faster depletion of synaptic resources. We will refer to this pattern of adaptation as type I adaptation (higher intensity more adaptation). Conversely, we have recently found that neurons along the lemniscal pathway do not adapt as predicted from STD. That is, increasing the intensity of stimulation caused less adaptation of lemniscal neurons (Ganmor et al., 2010). This includes the first station of this pathway, the brainstem Principalis nucleus $(\mathrm{PrV})$. We will refer to this unexpected pattern of adaptation as type II adaptation.

The other major somatosensory pathway, the paralemniscal pathway (Diamond et al., 1992), has not been investigated in the context of intensity-dependent adaptation. This pathway emerges from the distinct brainstem Interpolaris nucleus (SpVi). Despite their spatial segregation, the two pathways interact at the brainstem level (Furuta et al., 2008). Specifically, inputs from $\mathrm{SpVi}$ neurons are responsible for the synthesis of multiwhisker receptive fields (RFs) in the PrV (Timofeeva et al., 2004). It was proposed that the two pathways also have different roles in active sensation (Yu et al., 2006). Whereas the paralemniscal pathway conveys sensor motion (whisking) signals, the lemniscal pathway conveys whisker-touch signals. However, other studies found that the firing of thalamic paralemniscal neurons in awake animals is not correlated with whisking kinematics (Masri et al., 2008).

In this study, we have used in vivo intracellular and extracellular recordings to investigate the adaptation processes in these pathways at the brainstem level. We found that increasing the 
intensity of stimulation exerted opposite effects on the degree of adaptation in the two trigeminal nuclei. Moreover, the adaptation pattern to adjacent whisker (AW) stimulation in the $\operatorname{PrV}$ is similar to that of SpVi neurons, indicating that its receptive field profile strongly depends both on the intensity of stimulation and the progress of adaptation.

\section{Materials and Methods}

Animals. Animal surgeries and in vivo recordings were performed as previously described (Katz et al., 2006). Briefly, recordings were made from young adult Wistar rats of either sex (6-10 weeks old). After initial anesthesia with ketamine and xylazine (100 and $10 \mathrm{mg} / \mathrm{kg}$, i.p., respectively; Ketaset, Fort Dodge Animal Health), a tracheotomy was performed, and rats were mounted in a stereotaxic device and respired with a mixture of isoflurane $(0.5-1.5 \%)$ or halothane $(0.25-1 \%)$ and oxygenenriched air. End-tidal $\mathrm{CO}_{2}$ levels, heart rate (200-400 beats/min), and body temperature $\left(37^{\circ} \mathrm{C}\right)$ were monitored throughout the experiment. All surgical and experimental procedures were approved by the Weizmann Institute of Science Institutional Animal Care and Use Committee.

Brainstem recordings. To reach the brainstem trigeminal complex, we exposed the dorsal part of the cerebellum and the first cervical segment of the spinal cord. The dura was removed, and the area was continuously washed with ACSF or covered with warm agar (3\% in $0.9 \%$ saline). Sharp glass electrodes (borosilicate: outer diameter, $1.5 \mathrm{~mm}$; inner diameter, $0.86 \mathrm{~mm}$ ), pulled with a P-97 micropipette puller (Sutter Instruments), were filled with $2 \mathrm{M} \mathrm{K}$-acetate (resistance, $60-100 \mathrm{M} \Omega$ ), and in a subset of cells $(n=26) 20 \mathrm{~mm}$ QX-314 was added to prevent firing. Electrodes were inserted 2-3 mm lateral to the midline and $1 \mathrm{~mm}$ dorsal to the meeting point of the brainstem and cerebellum. Using a motorized manipulator (MX7600, Siskiyou), the electrode was advanced with an angle of $2-7^{\circ}$ horizontally and parallel to the sagittal plane. Intracellular signals were acquired using an Axoclamp-2B amplifier (Molecular Devices) low-pass filtered at $3 \mathrm{kHz}$ before being digitized at $20 \mathrm{kHz}$ using acquisition hardware and software (National Instruments). In some recordings, heart artifacts ( $\sim 4 \mathrm{~Hz}$ high pass) were filtered. Extracellular recordings were made using a broken sharp pipette filled with $2 \mathrm{~m} \mathrm{~K}$-acetate with a diameter of 5-10 $\mu \mathrm{m}$. Extracellular activity was acquired using an Axoclamp900A amplifier (Molecular Devices; gain, $100 \mathrm{~Hz}$; bandpass, $300 \mathrm{~Hz}$ to 3 $\mathrm{kHz}$ ) and digitized at $20 \mathrm{kHz}$ (National Instruments). The recording location was marked for histological identification with application of DiI on the external surface of the electrode.

Histology. Procedures were modified from the technique previously described (Katz et al., 2006). Briefly, the animal was overanesthetized, and perfused transcardially with PBS and heparin followed by $2.5 \%$ paraformaldehyde. Brains were removed and postfixed in the perfusion solution for $24 \mathrm{~h}$, in $30 \%$ sucrose for $24 \mathrm{~h}$, and were cut in a microtome (SM 2000R, Leica). Slices were imaged in bright-field and epifluorescence modes using a Leica DFC365-FX camera.

Whisker stimulation. Whiskers were trimmed to $10-20 \mathrm{~mm}$. A single whisker was inserted into galvanometer servo control motor with a matching servo driver and controller $(6210 \mathrm{H}$, MicroMax 677xx, Cambridge Technology Inc.). The displacement was measured off-line using an optical displacement measuring system (optoNCDT 1605, MicroEpsilon), indicating that ringing was negligible. A fast-rising voltage command was used to evoke a fast whisker deflection with a constant rise time of $1 \mathrm{~ms}$ followed by a $20 \mathrm{~ms}$ ramp-down signal. Amplitude and speed of deflection grow together with the magnitude of the voltage command, following a quasilinear relationship, as we verified that both affect the adaptation profile in the same direction (data not shown). In each recording session, two stimulus intensities were used. Stimulation velocity at the high-intensity condition was $\sim 50 \mathrm{~mm} / \mathrm{s}(\sim 145 \mu \mathrm{m}$ displacement). For the weak stimulus condition, stimulation was set such that it evoked a response with at least $10 \%$ lower subthreshold postsynaptic potential (PSP) amplitude or firing probability. A train of 20 stimuli at $18 \mathrm{~Hz}$ was applied at each trial (20 to 40 trials for each intensity). Intertrial intervals were $3 \mathrm{~s}$, and trials were pseudo-randomized.

Statistical analysis. The statistical analyses for the populations were conducted using two-way ANOVA followed by Tukey's HSD post hoc tests. Each cell was analyzed for the type of adaptation using the Wilcoxon two-sided rank sum test. The significance level is at least $p<0.05$, and results are reported as the mean \pm SE unless otherwise stated.

\section{Results}

Our previous extracellular recording study of neurons in the $\mathrm{PrV}$, the first station of the lemniscal pathway, showed a peculiar form of intensity-dependent adaptation (Ganmor et al., 2010), where increasing the intensity of stimulation resulted in less adaptation. We used intracellular recordings to find whether this adaptation pattern results from the properties of the synaptic inputs or from the transformation into spikes. The principal whisker (PW) was stimulated with two different intensities of repetitive whisker stimulation. Similar to their firing response and in contrast to predictions of the STD model, the PSPs of PrV neurons adapted less when the intensity of whisker stimulation was increased (Fig. $1 A$, example cell). For this cell, the initial response to the higher intensity was larger by $21 \pm 1.5 \%$ when compared with the weaker intensity. Despite that, the cell adapted less to the higherintensity stimulation. This is clearly observed in the normalized response curves (Fig. $1 B$ ) obtained from the averaged peak responses in individual trials. We will denote this adaptation profile as type II.

Although we previously showed that adaptation of neurons in the trigeminal ganglion is weak and is intensity independent (Ganmor et al., 2010), type II adaptation may arise from the mechanical properties of the whiskers and thus be present in other nuclei of the trigeminal complex. Hence, we examined the intensity-dependent adaptation of SpVi neurons. Since the recording electrodes were inserted in a rostrocaudal trajectory, parallel to the longitudinal axis of the trigeminal complex, the locations of the recorded cells in the two nuclei differed substantially based on the penetration depth (Fig. $1 C-E$ ). In contrast to $\mathrm{PrV}$ neurons, SpVi neurons adapted as predicted from STD, namely, high-intensity stimulation led to more adaptation when compared with low-intensity stimulation (Fig. $1 F, G$ ). We will denote this adaptation profile as type I.

Importantly, type II adaptation was independent of the exact mechanical properties of stimulation. Increasing either the amplitude or the velocity of whisker deflection entailed less adaptation in PrV neurons. In addition, we found a monotonically inverse relationship between $\operatorname{PrV}$ adaptation and four increasing levels of intensity, suggesting that ceiling effects are also not responsible for this adaptation pattern. Furthermore, type II adaptation was independent of the exact frequency of the adapting train. These findings (data not shown) suggest that the exact mechanical properties of the stimulus were not responsible for type II adaptation. Finally, whisker stimulation intensities were similar in both nuclei (PrV: low, $22 \pm 3.7 \mathrm{~mm} / \mathrm{s}$; high, $48.8 \pm 5.4$ $\mathrm{mm} / \mathrm{s}$; SpVi: low, $19.1 \pm 4.6 \mathrm{~mm} / \mathrm{s}$; high, $49.3 \pm 8.3 \mathrm{~mm} / \mathrm{s}$ ).

Intracellularly recorded neurons $(n=33)$ were classified by their adaptation profiles (type I/II) and clustered into two groups by the depth of penetration (Paxinos, 2007). Most PrV neurons displayed type II adaptation (Fig. $2 A$, left; depth, $3.71 \pm 0.19 \mathrm{~mm}$; type II, $n=15$; type I, $n=3$ ), whereas the typical adaptation pattern of SpVi neurons was of type I (Fig. $2 A$, right; depth, $2.1 \pm$ $0.11 \mathrm{~mm}$; type II, $n=3$; type I, $n=12$ ). The first response latency was similar in both nuclei $(\mathrm{PrV}, 2.70 \pm 0.04 \mathrm{~ms}$; SpVi, $2.72 \pm 0.06$ $\mathrm{ms}$ ), as expected from the monosynaptic innervations from the trigeminal ganglion. Additionally, there was no apparent separation in the SpVi regarding its caudal and rostral parts (Pierret et al., 2000), as the difference between high- and low-intensity stimulation was not depth dependent (data not shown). 

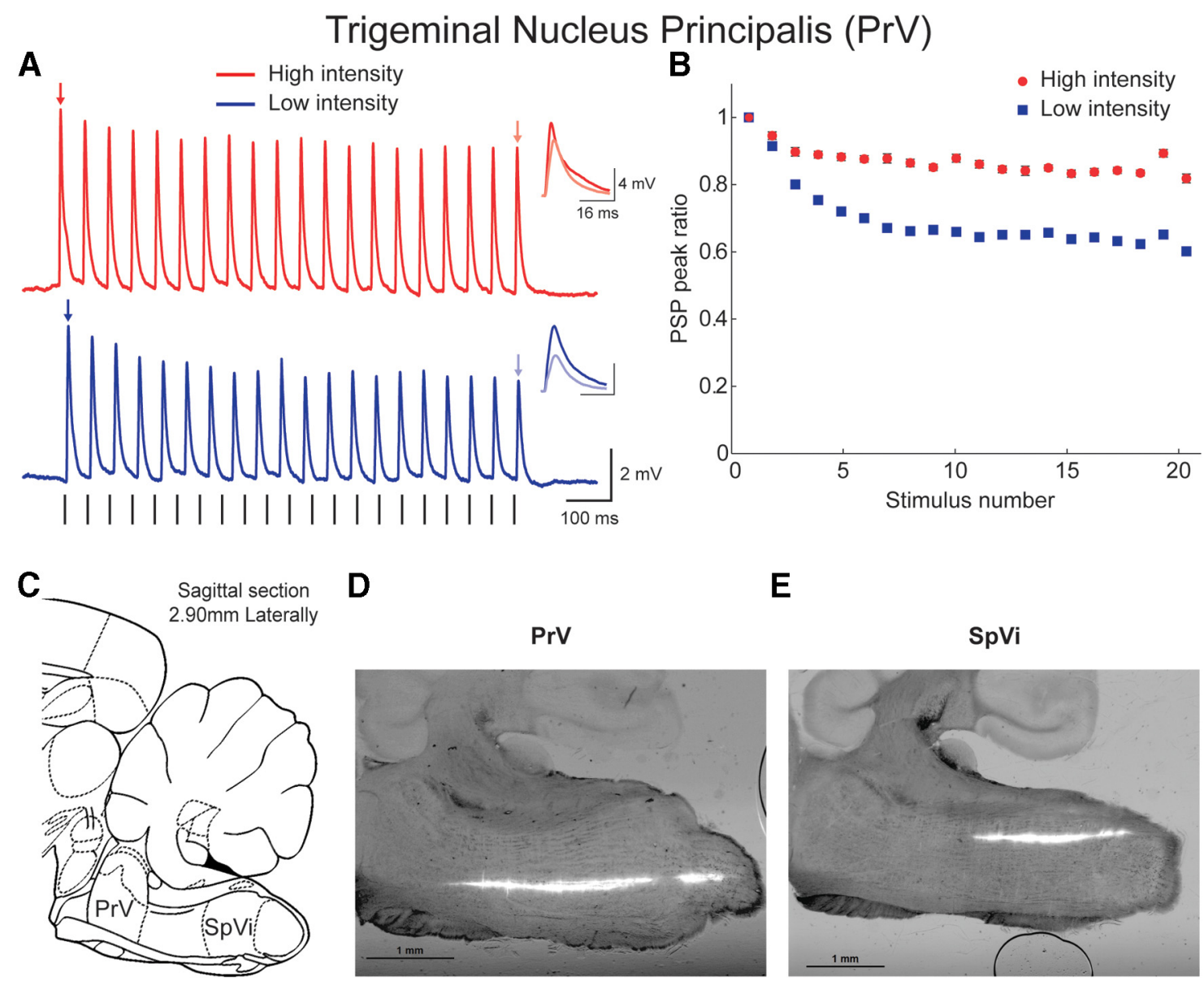

D $\quad$ E

E

PrV

$\mathrm{SpVi}$
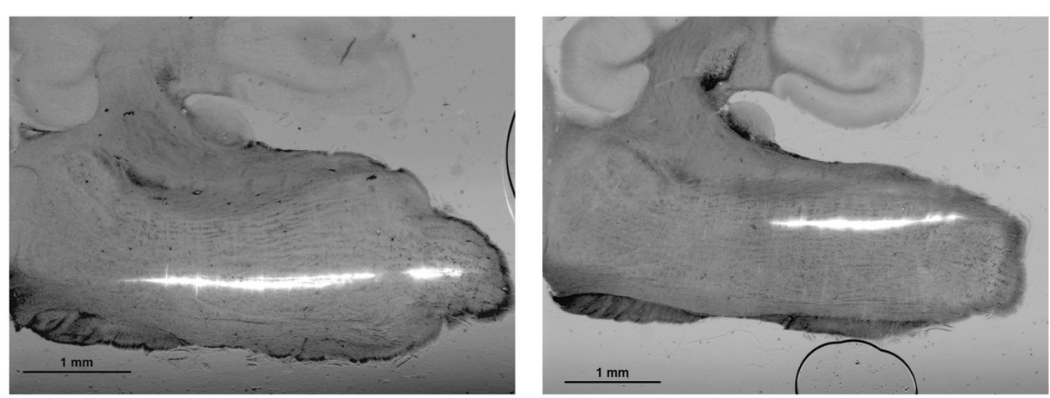

Trigeminal Nucleus Interpolaris (SpVi)

$\mathbf{F}$
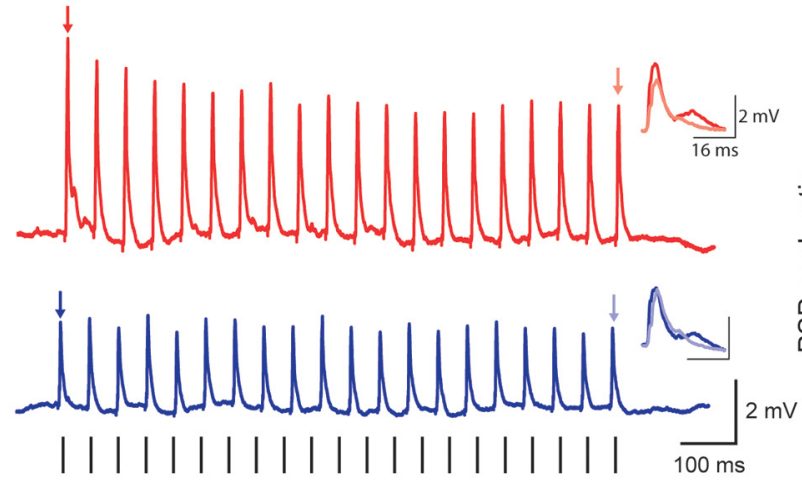

G

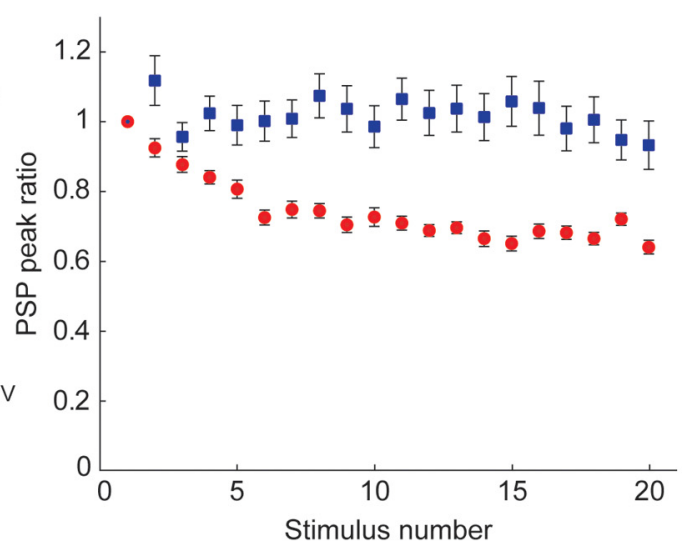

Figure 1. Adaptation in PrV and SpVi example cells. $A$, Average membrane potential of a PrV neuron recorded at a depth of $3615 \mu \mathrm{m}$ (resting potential, $-60.6 \mathrm{mV}$ ). The PW was stimulated 20 times at $18 \mathrm{~Hz}$ (black bars), at either high (red) or low (blue) intensity. $\boldsymbol{B}$, Average peak PSPs in $\boldsymbol{A}$ normalized to the first PSP (high, red circles; low, blue squares). Each data point was calculated by measuring the peak PSPs in individual trials followed by averaging. The response to high-intensity stimulation adapts less despite the larger response to the first stimulus. $C$, Separation of the two nuclei. $\boldsymbol{D}, \boldsymbol{E}$, Examples of histological labeling made using broken sharp electrodes coated with Dil to the PrV (D) and SpVi (E). $\boldsymbol{F}, \boldsymbol{G}$, Same as $\boldsymbol{A}$ and $\boldsymbol{B}$ for an SpVi neuron recorded at a depth of 2436 $\mu \mathrm{m}$ (resting potential, $-52.5 \mathrm{mV}$ ). The response to high-intensity stimulation adapts more.

We found that, on average across the population, PrV neurons adapted significantly less when stimulated with high-intensity stimulation (Fig. $2 B$; adapted to $80.31 \pm 1.64 \%$ of the response to the first stimulus) in comparison with low-intensity stimulation $(69.48 \pm 1.64 \%)$. In this type of analysis, quantified by the ratio between the peak of the last five responses of the average PSP and that of the first response, a lower percentage signifies more adap- tation. In contrast, SpVi neurons (Fig. 2C) adapted significantly less to low-intensity stimulation $(93.55 \pm 1.79 \%)$ compared with the higher stimulation intensity (73.85 $\pm 1.79 \%$; two-way ANOVA, $p<0.001$ for PrV and SpVi).

To find how the transformation from synaptic inputs to spikes affects the adaptation patterns, we analyzed the firing in these two nuclei. Additional populations of neurons were recorded using 
A

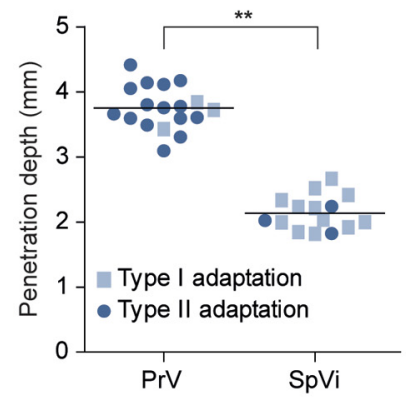

D

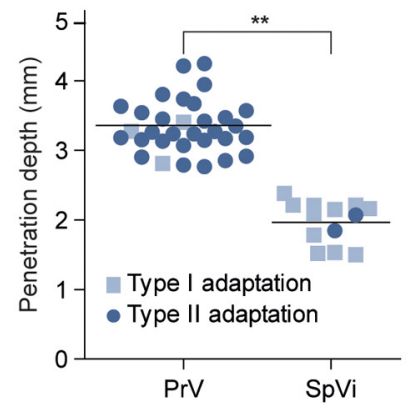

Subthreshold Responses

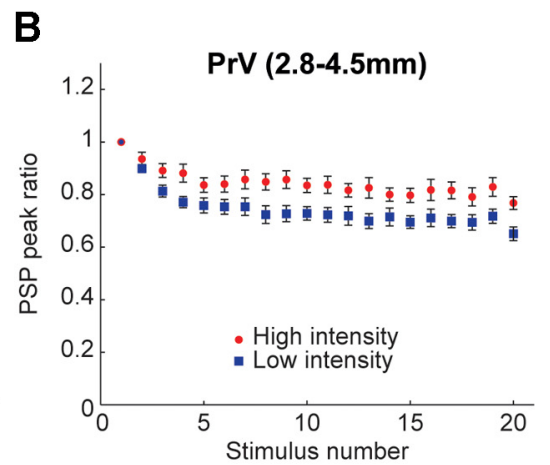

C

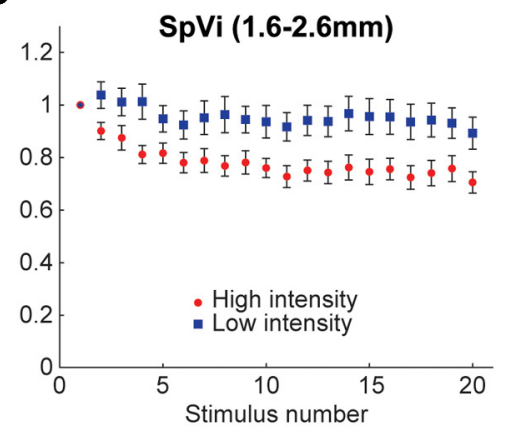

Suprathreshold Responses

E

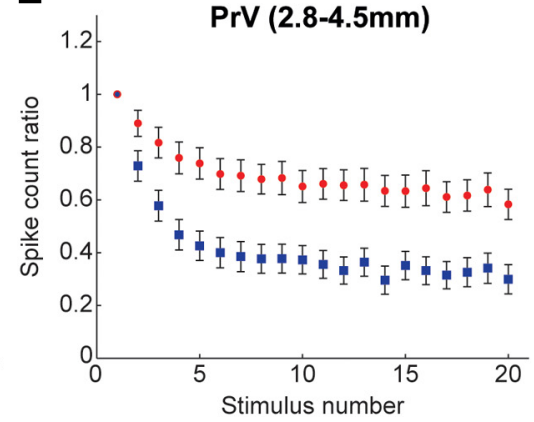

$\mathrm{F}$

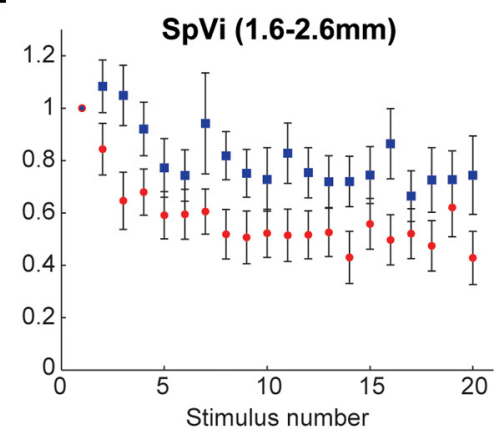

Figure 2. Adaptation patterns in PrV and SpVi are inversely dependent on tactile intensity. A, Penetration depth and adaptation type of all intracellularly recorded neurons. Type II, Cells demonstrating less adaption with increasing intensity of stimulation; type I, cells demonstrating more adaption with increasing intensity of stimulation. $\boldsymbol{B}$, Average response of 18 PrV neurons. Peak PSPs were normalized to the peak of the first response for each cell before the data were pulled and averaged across the population. Response to high-intensity stimulation adapted significantly less in comparison with the lower-intensity stimulation. C, Fifteen SpVi neurons: responses to the low-intensity stimulation adapted significantly less compared with the higher-intensity stimulation. $\boldsymbol{D}$, As in $\boldsymbol{A}$ for extracellularly recorded neurons. $\boldsymbol{E}, \boldsymbol{F}$, Spike data of $32 \mathrm{PrV}$ neurons and $13 \mathrm{SpVi}$ neurons: spike counts per stimulation were normalized for each cell to the first response.

broken sharp electrodes extracellularly or intracellularly without QX-314 (Fig. 2D). The PrV population was recorded at an average depth of $3.41 \pm 0.11 \mathrm{~mm}$ (type II, $n=29$; type I, $n=3$ ), and the $\mathrm{SpVi}$ population was recorded at an average depth of $1.90 \pm$ $0.09 \mathrm{~mm}$ (type II, $n=2$; type I, $n=11$ ). In congruence with our subthreshold results, firing of PrV neurons adapted significantly less to high-intensity stimulation $(62.11 \pm 2.4 \%)$ than to lowintensity stimulation (Fig. $2 E ; 32.75 \pm 2.4 \%$ ), while SpVi neurons exhibited the opposite pattern (Fig. $2 F$; high intensity, $51.65 \pm$ 4.2\%; low intensity, $74.51 \pm 4.2 \%$; two-way ANOVA, $p<0.001$ for $\mathrm{PrV}$ and $\mathrm{SpVi}$ ). The combined subthreshold and spike data show that the transformation into spikes amplifies the differences between the adaptations to high and low intensities when compared with the synaptic inputs.

Timofeeva et al. (2004) showed that inter-subnuclear interactions ( $\mathrm{SpVi}-\mathrm{PrV}$ ) shape the sizes of receptive fields in the PrV. Hence, we investigated how $\operatorname{PrV}$ neurons adapt in response to a repetitive stimulation of the AWs. In a subset of neurons, individual AWs were stimulated with the same two intensities as PWs. In accordance with previous extracellular recordings (Minnery and Simons, 2003), the AW response was weaker and had a longer latency (amplitude: PW, $7.05 \pm 0.75 \mathrm{mV}$; AW, $4.6 \pm 0.56$ mV; latency: PW, $2.7 \pm 0.1 \mathrm{~ms} ; \mathrm{AW}, 2.85 \pm 0.08 \mathrm{~ms} ; n=12$; Wilcoxon two-sided rank sum test, amplitude, $p<0.01$; latency, $p<0.05)$. Interestingly, while on average the PW response was of a type II adaptation profile (Fig. $3 A$; high intensity, $81.1 \pm 1.93 \%$; low intensity, $69.36 \pm 1.73 \%$ ), the response of the AWs in the same PrV cells was of a type I adaptation (Fig. $3 B$; high intensity, $58.21 \pm 1.83 \%$; low intensity, $68.17 \pm 1.96 \% ; n=12$; two-way
ANOVA: PW, $p<0.001$; AW, $p<0.05)$. That is, the adaptation profile of PrV neurons in response to AW stimulation demonstrated the same profile found for SpVi neurons (Fig. 2C). This suggests that the RF profile during adaptation of $\operatorname{PrV}$ neurons strongly depends on the intensity of stimulation, such that adaptation sharpens the RF, and this effect increases with higher stimulation intensity. Moreover, the coexistence of both types of adaptation indicates that intrinsic mechanisms are not involved in generating the intensity-dependent adaptation patterns.

Failure analysis of our intracellular data suggests that presynaptic mechanisms are partially involved. We analyzed the probability of observing a synaptic response for each stimulus in the train, which was considered a success if the peak amplitude was above noise level. Since a failure of a synaptic response reflects either a complete absence of presynaptic firing or a failure of synaptic transmission, this analysis can elucidate the source of the type II adaptation. Although the probabilities of observing a response were very high, a clear difference between intensities was found. The PSP probability curves to stimulation of the PW and AW (Fig. 3C,D) were similar in shape to the peak PSP curves (Fig. $3 A, B)$. When stimulating the PW, the PSP probability to lowintensity stimulation adapted to a significantly lower value $(88.5 \pm 2.06 \%)$ than that for the high-intensity stimulation (97.24 $\pm 2.06 \%$; Fig. $3 C$ ). On the other hand, stimulation of the AWs in the same set of PrV neurons showed more adaptation in the PSP probability to high-intensity stimulation (70.67 \pm $2.25 \%)$ than for the low-intensity stimulation $(84.84 \pm 1.52 \%$; Fig. 3D; two-way ANOVA: PW, $p<0.05$; AW, $p<0.01, n=12$ ). These results suggest that presynaptic mechanisms contribute to 


\section{Trigeminal Nucleus Principalis (PrV)}

A

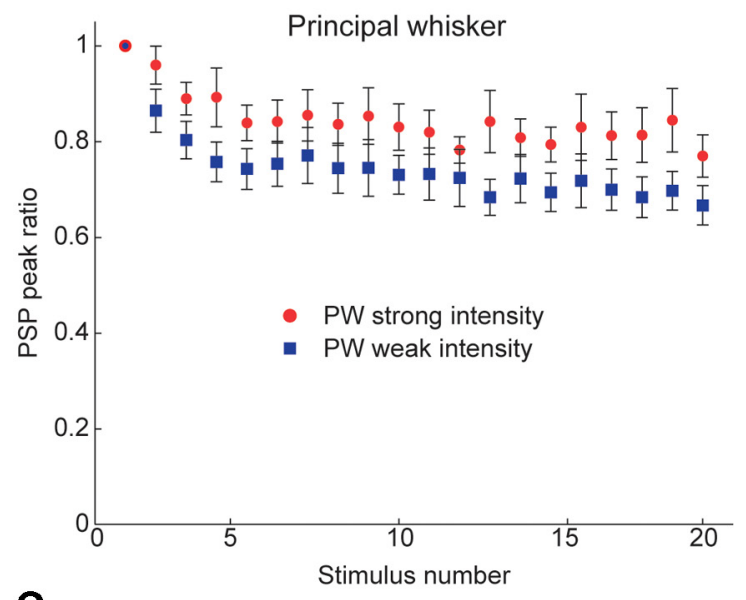

C

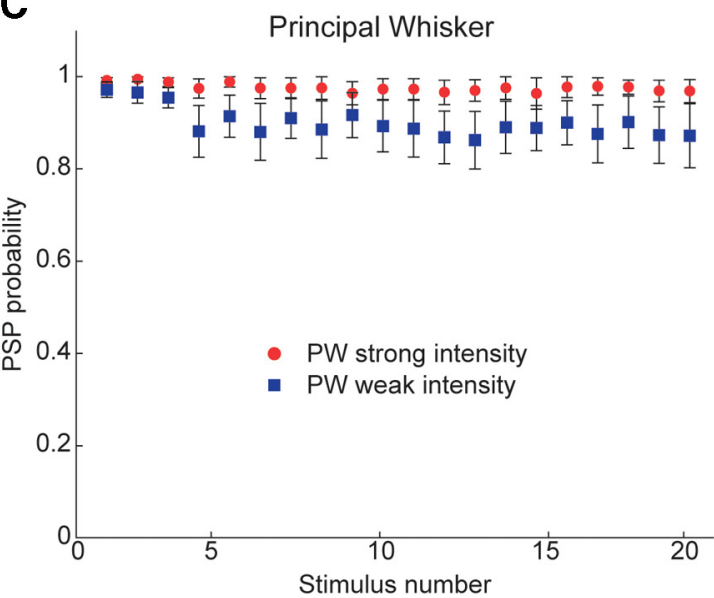

B

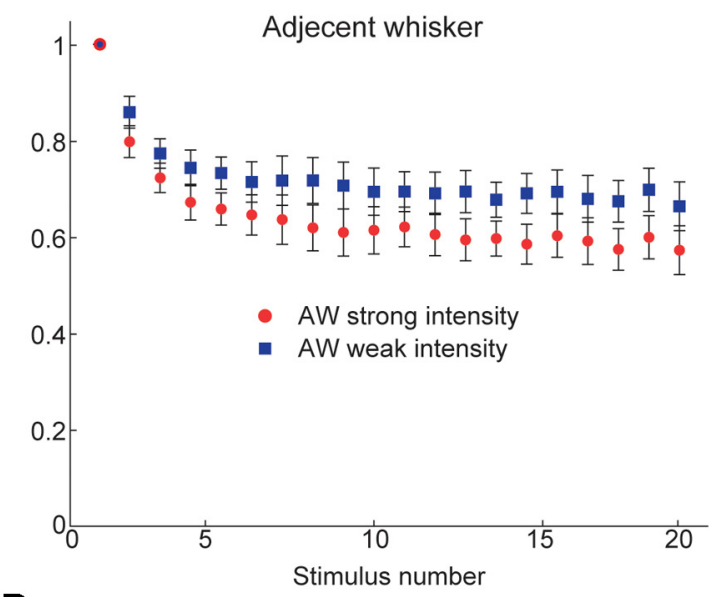

D

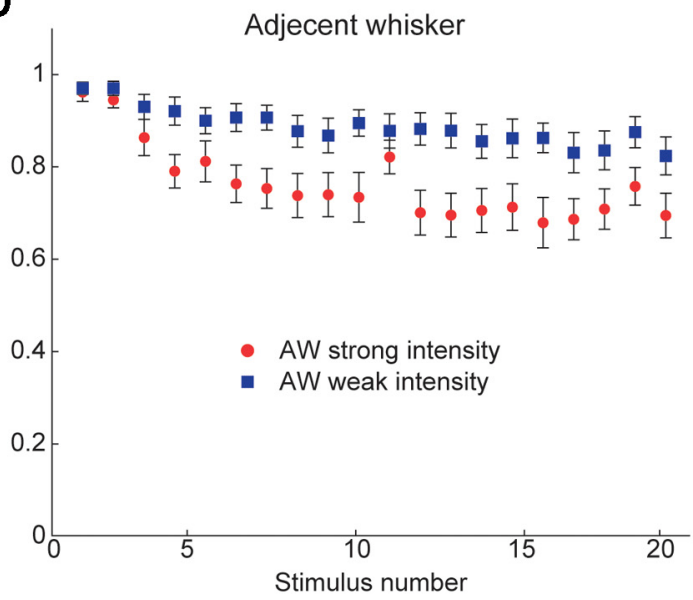

Figure 3. Intensity-dependent adaptation to AW stimulation of PrV neurons. $A$, The average normalized response of a subset of cells $(n=12)$ to high-and low-intensity stimulation of the PW. The response to high-intensity stimulation adapted significantly less than low-intensity stimulation. $\boldsymbol{B}$, The average normalized responses of the same set of cells to identical AW deflections. The response to low-intensity stimulation adapted significantly less than high-intensity stimulation. C, PSP probability analysis for the responses in $A$. The probability of observing a PSP to low-intensity stimulation adapted significantly more than that for the high-intensity stimulation. D, PSP probability analysis for the stimulated AWs of the same set of PrV neurons showed more adaptation for the high-intensity stimulation than for the low-intensity stimulation.

the intensity-dependent adaptation patterns that distinguish between the response to PW and AW stimulation.

\section{Discussion}

Our results show distinct and opposite adaptation patterns in two major somatosensory brainstem nuclei. In the PrV, the first station of the lemniscal pathway, increasing the intensity of repetitive tactile stimulation entails less adaptation. In contrast, in the $\mathrm{SpVi}$, the first station of the paralemniscal pathway, increasing the intensity of stimulation results in more adaptation. Additionally, we found that stimulation of adjacent whiskers in the PrV results in the same adaptation pattern of SpVi neurons. Importantly, analysis of synaptic input probability of PrV neurons during train stimulation suggests that presynaptic mechanisms may contribute to intensity-dependent adaptation patterns in the $\mathrm{PrV}$. While the pattern of adaptation observed in the SpVi is expected from STD, the mechanism underlying the adaptation pattern of PrV neurons remains unclear. We suggest that these pathways evolved to convey sustained sensory inputs of very different dynamic ranges.

To the best of our knowledge, this study is the first in which a large population of trigeminal complex neurons were recorded intracellularly in vivo. In agreement with the extracellular recording of Ganmor et al. (2010), we show that high-intensity repetitive whisker stimulation entails less adaptation in the firing output of the PrV compared with low-intensity stimulation (Fig. $2 E)$. Our current study directly shows that this pattern of adaptation already exists at the synaptic level. In addition, the mean response latency to mechanical stimulation of a single whisker, $\mathrm{RF}$ size, and the somatotopic organization of PrV and SpVi neurons unveiled using intracellular recordings are comparable to previous extracellular recording studies (Lo et al., 1999; Minnery and Simons, 2003).

Several mechanisms may account for the unexpected pattern of synaptic adaptation in the PrV (i.e., type II). First, it is possible that the type I adaptation pattern in combination with a threshold effect (i.e., iceberg effect) accounts for the type II adaptation pattern. According to this hypothesis, although weak synaptic inputs barely adapt, they rapidly become too small to cross the threshold; whereas stronger inputs remain above threshold throughout the train. However, that the adaptation pattern of intracellularly recorded PrV neurons is observed at subthreshold inputs indicates that this phenomenon is of subthreshold origin 
and not purely a nonlinearity in the PSP to spike transformation. Other possible mechanisms include the mechanical properties of the whisker follicle, intrinsic nonsynaptic mechanisms, and unconventional synaptic depression dynamics of ascending inputs from the trigeminal ganglion. Another valid explanation might be that high-intensity stimulation recruits different fibers with different synaptic properties. There is no anatomical or functional evidence to support this hypothesis; however, our current results cannot revoke this theory. Finally, the unexpected pattern of adaptation may depend on presynaptic feedback inputs from local or remote circuits.

Mechanical and intrinsic mechanisms are unlikely to contribute to the pattern of intensity-dependent adaptation of PrV cells. First, the two nuclei demonstrate inverse relations between intensity and adaptation when using the same type of mechanical stimulation. Second, intensity-dependent adaptation of PrV cells upon stimulation of AWs is opposite to that of PW stimulation, indicating that the adaptation patterns are not caused by mechanical properties of the whisker follicle or due to intrinsic mechanisms. Network interactions in the trigeminal complex may also explain the peculiar type II adaptation of PrV neurons. Since SpVi neurons provide inhibitory inputs to the PrV (Furuta et al., 2008), as the intensity of stimulation increases inhibitory neurons of the SpVi adapt more, a process that leads to more disinhibition of PrV cells and consequently to less adaptation.

Although the possibility that SpVi neurons shape the adaptation of PrV neurons requires further investigation, the pattern of intensity-dependent adaptation of $\mathrm{PrV}$ neurons upon AW stimulation strongly supports it. Timofeeva et al. (2004) demonstrated the functional role of the inter-subnuclear connections in the synthesis of multiwhisker receptive fields in the PrV by SpVi neurons. This finding led us to assume that SpVi affects the adaptation pattern of PrV neurons when the AW is stimulated. Remarkably, the adaptation of $\mathrm{PrV}$ neurons in response to AWs stimulation is of the same type as for SpVi neurons (Fig. $3 B$ ), strongly suggesting that whisker-specific connections exist among these nuclei. While sharpening of receptive field size was reported in previous studies (Moore, 2004; Heiss et al., 2008), our study shows that a PrV neuron will adapt less to high-intensity stimulation if the PW is stimulated and will adapt more to the same change in intensity if the AW is stimulated. This may have a functional role in controlling the responsiveness and size of the RF as a function of stimulus intensity during sustained stimulation.

Interestingly, synaptic failure analysis of evoked PSPs shows a similar adaptation pattern when compared with the average PSP of PrV cells for PW and AW stimulation (Fig. 3). That is, the probability of observing synaptic input when the PW is stimulated adapts less as the intensity increases and vice versa when the AW was stimulated. Hence, these results strongly suggest that presynaptic mechanisms differentially contribute to the adaptation patterns of PrV neurons upon stimulation of the PW and AWs. Previous anatomical studies indeed demonstrated that the GABAergic inhibitory connections between the SpVi and $\mathrm{PrV}$ are mainly presynaptic (Yu and Avery, 1974; Bae et al., 2000). Hence, these studies strongly support our hypothesis that the SpVi shapes the intensity-dependent adaptation observed for PrV cells.

In our earlier work (Ganmor et al., 2010), we demonstrated that type II adaptation pattern is preserved along the lemniscal pathway, from the brainstem via the ventral posterior medial nucleus to cortical layer 4 . Yet, it remains to be determined whether type I adaptation is also preserved in higher stations of the paralemniscal pathway, in the posterior medial (POm) nucleus and in L5a of S1. However, as the POm nucleus is under inhibition in the anesthetized animal (Trageser et al., 2006), experiments in awake animals are necessary.

While the role of parallel adapting pathways is not fully understood, recent studies suggested that these channels may optimize the encoding of sensory inputs varying in time and amplitude (Kastner and Baccus, 2011; McGillivray et al., 2012). Indeed, our study indicates that the two main pathways of the vibrissa system demonstrate opposite intensity-dependent adaptation properties. Together, these pathways may enhance the throughput of information downstream to the cortex when conveying sustained inputs of different dynamic ranges.

\section{References}

Baccus SA, Meister M (2002) Fast and slow contrast adaptation in retinal circuitry. Neuron 36:909-919. CrossRef Medline

Bae YC, Ihn HJ, Park MJ, Ottersen OP, Moritani M, Yoshida A, Shigenaga Y (2000) Identification of signal substances in synapses made between primary afferents and their associated axon terminals in the rat trigeminal sensory nuclei. J Comp Neurol 418:299-309. CrossRef Medline

Chung S, Li X, Nelson SB (2002) Short-term depression at thalamocortical synapses contributes to rapid adaptation of cortical sensory responses in vivo. Neuron 34:437-446. CrossRef Medline

Dalton P (2000) Psychophysical and behavioral characteristics of olfactory adaptation. Chem Senses 25:487-492. CrossRef Medline

Diamond ME, Armstrong-James M, Ebner FF (1992) Somatic sensory responses in the rostral sector of the posterior group (POm) and in the ventral posterior medial nucleus (VPM) of the rat thalamus. J Comp Neurol 318:462-476. CrossRef Medline

Fairhall AL, Lewen GD, Bialek W, de Ruyter Van Steveninck RR (2001) Efficiency and ambiguity in an adaptive neural code. Nature 412:787-792. CrossRef Medline

Furuta T, Timofeeva E, Nakamura K, Okamoto-Furuta K, Togo M, Kaneko T, Deschênes M (2008) Inhibitory gating of vibrissal inputs in the brainstem. J Neurosci 28:1789-1797. CrossRef Medline

Ganmor E, Katz Y, Lampl I (2010) Intensity-dependent adaptation of cortical and thalamic neurons is controlled by brainstem circuits of the sensory pathway. Neuron 66:273-286. CrossRef Medline

Grill-Spector K, Henson R, Martin A (2006) Repetition and the brain: neural models of stimulus-specific effects. Trends Cogn Sci 10:14-23. CrossRef Medline

Hansen BJ, Dragoi V (2011) Adaptation-induced synchronization in laminar cortical circuits. Proc Natl Acad Sci U S A 108:10720-10725. CrossRef Medline

Heiss JE, Katz Y, Ganmor E, Lampl I (2008) Shift in the balance between excitation and inhibition during sensory adaptation of S1 neurons. J Neurosci 28:13320-13330. CrossRef Medline

Kastner DB, Baccus SA (2011) Coordinated dynamic encoding in the retina using opposing forms of plasticity. Nat Neurosci 14:1317-1322. CrossRef Medline

Katz Y, Heiss JE, Lampl I (2006) Cross-whisker adaptation of neurons in the rat barrel cortex. J Neurosci 26:13363-13372. CrossRef Medline

Lo FS, Guido W, Erzurumlu RS (1999) Electrophysiological properties and synaptic responses of cells in the trigeminal principal sensory nucleus of postnatal rats. J Neurophysiol 82:2765-2775. Medline

Masri R, Bezdudnaya T, Trageser JC, Keller A (2008) Encoding of stimulus frequency and sensor motion in the posterior medial thalamic nucleus. J Neurophysiol 100:681-689. CrossRef Medline

McGillivray P, Vonderschen K, Fortune ES, Chacron MJ (2012) Parallel coding of first- and second-order stimulus attributes by midbrain electrosensory neurons. J Neurosci 32:5510-5524. CrossRef Medline

Minnery BS, Simons DJ (2003) Response properties of whisker-associated trigeminothalamic neurons in rat nucleus principalis. J Neurophysiol 89: 40-56. CrossRef Medline

Moore CI (2004) Frequency-dependent processing in the vibrissa sensory system. J Neurophysiol 91:2390-2399. CrossRef Medline

Ohzawa I, Sclar G, Freeman RD (1982) Contrast gain control in the cat visual cortex. Nature 298:266-268. CrossRef Medline 
Paxinos G (2007) The rat brain in stereotaxic coordinates, Ed 6. Amsterdam: Elsevier.

Pierret T, Lavallée P, Deschênes M (2000) Parallel streams for the relay of vibrissal information through thalamic barreloids. J Neurosci 20:74557462. Medline

Schweinberger SR, Casper C, Hauthal N, Kaufmann JM, Kawahara H, Kloth N, Robertson DM, Simpson AP, Zäske R (2008) Auditory adaptation in voice perception. Curr Biol 18:684-688. CrossRef Medline

Timofeeva E, Lavallée P, Arsenault D, Deschênes M (2004) Synthesis of multiwhisker-receptive fields in subcortical stations of the vibrissa system. J Neurophysiol 91:1510-1515. CrossRef Medline

Trageser JC, Burke KA, Masri R, Li Y, Sellers L, Keller A (2006) Statedependent gating of sensory inputs by zona incerta. J Neurophysiol 96: 1456-1463. CrossRef Medline

Tsodyks MV, Markram H (1997) The neural code between neocortical py- ramidal neurons depends on neurotransmitter release probability. Proc Natl Acad Sci U S A 94:719-723. CrossRef Medline

Wang Q, Webber RM, Stanley GB (2010) Thalamic synchrony and the adaptive gating of information flow to cortex. Nat Neurosci 13: 1534-1541. CrossRef Medline

Wark B, Lundstrom BN, Fairhall A (2007) Sensory adaptation. Curr Opin Neurobiol 17:423-429. CrossRef Medline

Wilent WB, Contreras D (2004) Synaptic responses to whisker deflections in rat barrel cortex as a function of cortical layer and stimulus intensity. J Neurosci 24:3985-3998. CrossRef Medline

Yu C, Derdikman D, Haidarliu S, Ahissar E (2006) Parallel thalamic pathways for whisking and touch signals in the rat. PLoS Biol 4:e124. CrossRef Medline

Yu HH, Avery JK (1974) Primary afferent depolarization: direct evidence in the trigeminal system. Brain Res 75:328-333. CrossRef Medline 